\title{
Efficient modelling of contact interfaces of joints in built-up structures
}

\author{
L. Gaul ${ }^{1} \&$ M. Mayer ${ }^{2}$ \\ ${ }^{1}$ Institute of Applied and Experimental Mechanics, \\ University of Stuttgart, Germany \\ ${ }^{2}$ Engineering and Test Center - Numerical Simulation, \\ Gasoline Systems Division, Robert Bosch GmbH, Germany
}

\begin{abstract}
This paper introduces an improved approach to model contact interfaces of fixed joints in finite element analysis (FEA) with regard to prediction of the vibration behaviour of built-up structures. The approach consists of two parts: (1) the development of a suitable, new contact model which considers the most important physical effects of wave propagation over the contact interface; and (2) the implementation of the contact model in commercial finite element software. The new model is based on the contact models of Hertz and Mindlin for the contact of a single spherical asperity with an elastic plane. The Hertzian microscopic model for normal contact is then generalized with a statistical approach for rough surfaces introduced by Greenwood to a macroscopic normal contact model for engineering surfaces. To model the macroscopic tangential contact, a new model based on Mindlins approach is introduced which accounts accurately for microslip effects and considers the dependence of tangential contact behaviour on the normal pressure. For implementing the contact model in FEA, a special isoparametric contact element, the so-called zero thickness element, is programmed. The use of this element compared with existing contact algorithms has some major advantages with regard to the application of modelling the contact in fixed joints considered in this paper. The introduced approach is verified by simulating the vibration behaviour of a built-up structure and proving the prediction quality by comparing simulation results with experimental data.

Keywords: contact mechanics, contact elements, joints, joint modelling, microslip, contact damping, hysteresis model, evolution equation.
\end{abstract}




\section{Introduction}

For lightly damped, linear members of a structure, very good estimates of eigenfrequencies, modal damping values, and corresponding mode shapes can be achieved by Experimental Modal Analysis (EMA). Furthermore, by model updating of finite element models of members, very good predictions of the vibration behaviour up to high frequencies are possible [1].

If we now assemble single members into a built-up structure, prediction of the structural vibration behaviour can be quite involved, even though the behaviour of all single members is well-known [2]. This is due to the fact that the mechanical contact at joint interfaces is usually not modelled sufficiently. Effects like uneven contact pressure distributions over the contact area, microslip damping and gaping of contact regions remain unconsidered but these effects can have a major influence on the structural vibration behaviour.

To account for these effects, this paper shows that the roughness of contacting surfaces (figure 1) has to be considered at least integrally to predict the vibration behaviour of built-up structures meaning resonance frequencies, mode shapes and modal damping values.



Figure 1: Although the contact area seems to be conforming on a macroscopic scale, the true contact consists of a multitude of non-conforming asperity contacts on a microscopic scale.

\section{Contact mechanics}

\subsection{Normal contact of rough surfaces}

For describing the normal contact of two rough surfaces, the contact model of Greenwood and Williamson is employed. This model is based on the Hertzian normal contact model for two elastic spheres [3] which is used to model the contact of single asperities. The Hertzian model leads to a circular contact region with radius $r_{A}$ and a radial normal pressure distribution

$$
p_{\mathrm{N}}(r)=p_{\max } \sqrt{1-\frac{r^{2}}{r_{A}^{2}}} \quad \text { with } \quad p_{\max }=\frac{3 F_{\mathrm{N}}}{2 \pi r_{A}^{2}}
$$


caused by normal force $F_{\mathrm{N}}$. The approach $\alpha_{\mathrm{N}}$ of facing points in the spheres which are far away from the contact region is given by

$$
\alpha_{\mathrm{N}}=\frac{r_{A}^{2}}{r^{*}}=\left(\frac{9 F_{\mathrm{N}}^{2}}{16 r^{*} E^{* 2}}\right)^{1 / 3} .
$$

Based on this, Greenwood and Williamson [4] developed a model to describe the contact of a rough elastic with a planar rigid surface, see figure 2 . The height distribution $\phi(z)$ of the rough surface is determined by the height distributions of the two real rough surfaces in contact. The height distribution $\phi(z)$ and also the

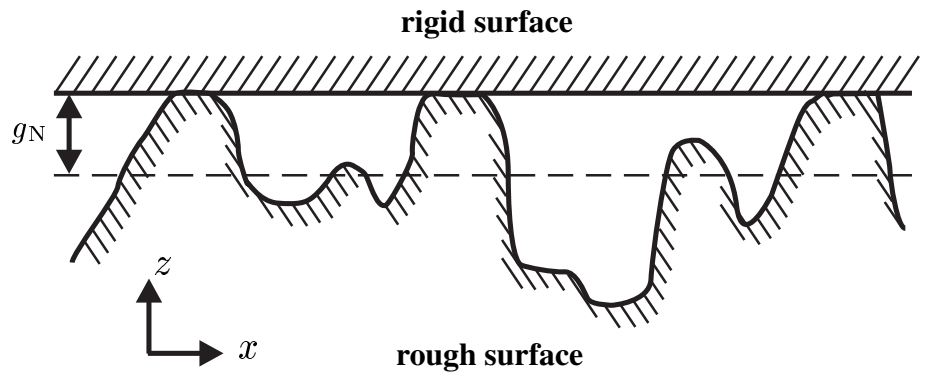

Figure 2: Contact of a planar rigid surface with a rough elastic surface. The dashed line represents the reference plane of the rough surface defined by the arithmetic mean value of the heights. $g_{\mathrm{N}}$ is the positive distance in normal direction between the rigid plane and the reference plane.

cumulative height distribution $\Phi(z)$ (Abbott-curve) can then be approximated by different distribution functions. For simplicity an exponential distribution function is used here:

$$
\phi_{\operatorname{Exp}}(z)=\sigma^{-1} e^{-(z / \sigma)} \quad \text { and } \quad \Phi_{\operatorname{Exp}}(z)=\left[-e^{-(\tilde{z} / \sigma)}\right]_{z}^{\infty}=e^{-(z / \sigma)} .
$$

This type of distribution is usually sufficient to describe the uppermost $25 \%$ of a measured cumulative height distribution [4] and leads to a relatively simple mathematical model. With this we get relations for the true area of contact $A_{\mathrm{R}}$ and the normal force $F_{\mathrm{N}}$ depending on the normal distance $g_{\mathrm{N}}$ of the two surfaces:

$$
\begin{gathered}
A_{\mathrm{R}}=\pi r_{\mathrm{A}} N \int_{g_{\mathrm{N}}}^{\infty}\left(z-g_{\mathrm{N}}\right) \sigma^{-1} e^{-z / \sigma} \mathrm{d} z=\pi r_{\mathrm{A}} N \sigma e^{-\left(g_{\mathrm{N}} / \sigma\right)}, \\
F_{\mathrm{N}}=\frac{4}{3} N E^{*} r_{\mathrm{A}}{ }^{1 / 2} \int_{g_{\mathrm{N}}}^{\infty}\left(z-g_{\mathrm{N}}\right)^{3 / 2} \sigma^{-1} e^{-z / \sigma} \mathrm{d} z=\pi^{1 / 2} N E^{*} r_{\mathrm{A}}^{1 / 2} \sigma^{3 / 2} e^{-g_{\mathrm{N}} / \sigma},
\end{gathered}
$$

where $\sigma$ is the standard deviation of the height profile of the rough surface, $N$ the overall number of peaks and $E^{*}$ the average Young's modulus. 
Normal pressure is obtained by dividing normal force by apparent area of contact $A_{0}$. By introducing new parameters the pressure can be expressed by [5]

$$
p_{\mathrm{N}}=F_{\mathrm{N}} / A_{0}=p_{\mathrm{N} 0} e^{-\lambda\left(g_{\mathrm{N}}-g_{\mathrm{N} 0}\right)} \quad \lambda=1 / \sigma>0,
$$

where $g_{\mathrm{N} 0}>0$ is the initial distance between the reference plane and the highest peak of the rough surface and $p_{\mathrm{N} 0}>0$ is the pressure value at initial contact $\left(g_{\mathrm{N}}=g_{\mathrm{N} 0}\right)$. The slope of this curve is given by $k_{\mathrm{N}}=-\lambda p_{\mathrm{N}}$ which corresponds to the normal contact stiffness. The asumption of an exponential height distribution leads to the interesting relation

$$
p_{\mathrm{N}} \sim A_{\mathrm{R}} \quad \text { with } \quad \frac{A_{\mathrm{R}}}{A_{0}}=\Phi\left(g_{\mathrm{N}}-g_{\mathrm{N} 0}\right)=e^{-\left(g_{\mathrm{N}}-g_{\mathrm{N} 0}\right) / \sigma} .
$$

\subsection{A new hysteresis model for tangential contact}

The introduced model is a new hysteresis model defined by an evolution equation which is based on Mindlin's approach for tangential contact of two spheres. Mindlin assumes that - by applying a tangential force $F_{\mathrm{T}}$ additionally to an acting normal force $F_{\mathrm{N}}$ on the spheres - contact area and normal pressure distribution remain the same as without a tangential force. Therefore, both variables can be described by Hertzian theory [3, chapter 7].

In the presliding or microslip regime $\left(0 \leq\left|F_{\mathrm{T}}\right|<\mu F_{\mathrm{N}}\right)$ the relation between tangential force $F_{\mathrm{T}}$ and relative tangential displacement $g_{\mathrm{T}}$ is defined by

$$
\left|F_{\mathrm{T}}\right|=\mu F_{\mathrm{N}}\left[1-\left(1-\left|g_{\mathrm{T}}\right| / g_{\mathrm{T}}^{\max }\right)^{3 / 2}\right] \quad \text { with } \quad g_{\mathrm{T}}^{\max }=\frac{3}{2 k_{\mathrm{T} 0}} \mu F_{\mathrm{N}},
$$

where $k_{\mathrm{T} 0}$ is the slope of the microslip curve at the origin and $g_{\mathrm{T}}$ is the relative tangential displacement of facing points in the spheres which are far away from the contact region. If $\left|F_{\mathrm{T}}\right|$ exceeds $\mu F_{\mathrm{N}}$ the whole contact area is slipping under the condition of Coulomb's law.

By differentiating equation (8) with respect to time where $F_{\mathrm{N}}$ is held constant we get the evolution equation

$$
\dot{F}_{\mathrm{T}}=k_{\mathrm{T} 0} \dot{g}_{\mathrm{T}}\left(1-\frac{\left|g_{\mathrm{T}}\right|}{g_{\mathrm{T}}^{\max }}\right)^{1 / 2}=k_{\mathrm{T} 0} \dot{g}_{\mathrm{T}}\left(1-\frac{\left|F_{\mathrm{T}}\right|}{\mu F_{\mathrm{N}}}\right)^{1 / 3} .
$$

This equation can be generalized by introducing an arbitrary exponent $n$ which can be used to adapt the curve to measured data, see figure 3 :

$$
\dot{F}_{\mathrm{T}}=k_{\mathrm{T} 0} \dot{g}_{\mathrm{T}}\left(1-\frac{\left|F_{\mathrm{T}}\right|}{\mu F_{\mathrm{N}}}\right)^{n} \quad \text { for } \quad 0 \leq\left|F_{\mathrm{T}}\right|<\mu F_{\mathrm{N}}, \quad n \in(0,1) .
$$

The slope of this curve is given by $k_{\mathrm{T}}=\mathrm{d} F_{\mathrm{T}} / \mathrm{d} g_{\mathrm{T}}=\dot{F}_{\mathrm{T}} / \dot{g}_{\mathrm{T}}$ which corresponds to the tangential contact stiffness. This evolution equation is valid for increasing tangential loading. For decreasing $\left|F_{\mathrm{T}}\right|$ the relation between tangential force and tangential relative displacement is assumed to follow a linear elastic 


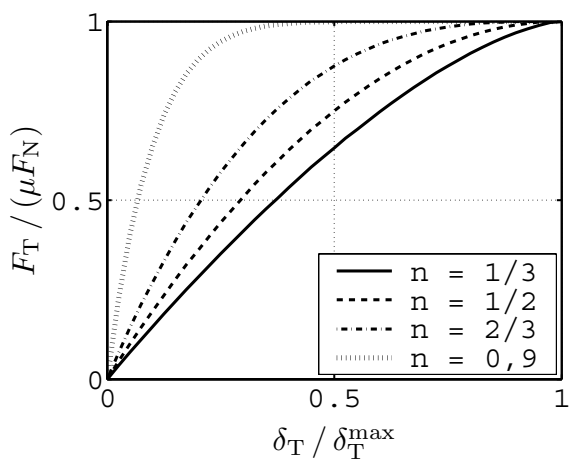

Figure 3: Hysteresis curve defined by equation (10) with different exponents $n$.

law, $\dot{F}_{\mathrm{T}}=k_{\mathrm{T} 0} \quad \dot{g}_{\mathrm{T}}$. The dissipated energy per cycle for an oscillating tangential force of constant amplitude $\hat{F}_{\mathrm{T}}$ and corresponding displacement amplitude $\hat{g}_{\mathrm{T}}$ is given by

$$
\begin{gathered}
W_{\mathrm{D}}=\frac{2 \mu^{2}{F_{\mathrm{N}}}^{2}}{k_{\mathrm{T} 0}}\left\{\frac{3}{2} \frac{\hat{g}_{\mathrm{T}}}{g_{\mathrm{T}}^{\max }}+\frac{3}{2} \frac{1-n}{2-n}\left[\left(1-\frac{\hat{g}_{\mathrm{T}}}{g_{\mathrm{T}}^{\max }}\right)^{(2-n) /(1-n)}-1\right]\right. \\
\left.-\frac{1}{2}\left[1-\left(1-\frac{\hat{g}_{\mathrm{T}}}{g_{\mathrm{T}}^{\max }}\right)^{3 / 2}\right]^{2}\right\}
\end{gathered}
$$

if the force amplitude is less than $\mu F_{\mathrm{N}}$. Otherwise, equation (11) has to be evaluated for $\hat{g}_{\mathrm{T}}=g_{\mathrm{T}}^{\max }$ and, additionally, the energy dissipated by Coulomb friction has to be considered:

$$
W_{\mathrm{D}}=\frac{2 \mu^{2} F_{\mathrm{N}}^{2}}{k_{\mathrm{T} 0}}\left[\frac{n(3-n)}{2\left(2-3 n+n^{2}\right)}+\frac{3}{2}\left(\frac{\hat{g}_{\mathrm{T}}}{g_{\mathrm{T}}^{\max }}-1\right)\right] .
$$

\section{Implementation of the contact model in FEA}

The concept of zero thickness elements goes back to Goodman et al. [6] and is discussed in detail in Hohberg [7]. A zero thickness element is depicted in figure 4 . The element consists of two four node quadrilateral elements which face each other. In each quadrilateral element, the three-dimensional displacement field $\{u\}=[u v v]^{T}$ is approximated by

$$
\left\{\begin{array}{c}
u \\
v \\
w
\end{array}\right\}=\left[\begin{array}{llll}
h_{1}[I] & h_{2}[I] & h_{3}[I] & h_{4}[I]
\end{array}\right]\left\{\begin{array}{l}
\left\{u_{1}\right\} \\
\left\{u_{2}\right\} \\
\left\{u_{3}\right\} \\
\left\{u_{4}\right\}
\end{array}\right\}=[H]\{u\}_{\text {nodal }},
$$


where $\left\{u_{i}\right\}=\left[\begin{array}{lll}u_{i} & v_{i} & w_{i}\end{array}\right]^{T}$ is the displacement vector of node $i$ and $h_{i}$ are the bilinear shape functions

$$
\begin{array}{ll}
h_{1}(\xi, \eta)=\frac{1}{4}(1-\xi)(1-\eta) & h_{3}(\xi, \eta)=\frac{1}{4}(1+\xi)(1-\eta) \\
h_{2}(\xi, \eta)=\frac{1}{4}(1-\xi)(1+\eta) & h_{4}(\xi, \eta)=\frac{1}{4}(1+\xi)(1+\eta)
\end{array}
$$

formulated in the natural coordinates $\xi, \eta$ of the element [8]. The matrix $[H]$ contains the shape functions and $[I]$ is a $3 \times 3$ unit matrix. The choice of a natural coordinate system simplifies numerical integration of the element matrices.

Distinguishing between top and bottom quadrilateral and assuming that each quadrilateral is connected to the surface of the finite element mesh of one contacting body, we denote the corresponding displacement fields of the elements as

$$
\{u\}^{\text {bottom }}=[H(\xi, \eta)]\{u\}_{\text {nodal }}^{\text {bottom }} \quad\{u\}^{\text {top }}=[H(\xi, \eta)]\{u\}_{\text {nodal }}^{\text {top }}
$$

Since these elements are only two-dimensional, the traction vector in each element

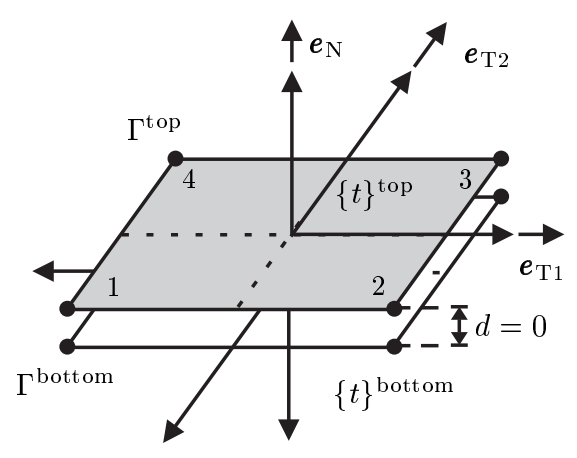

Figure 4: 8-node zero thickness element consisting of two 4-node quadrilateral elements which are connected to the surfaces of the contacting bodies.

describes the interface stresses

$$
\{t\}^{\text {bottom }}=\left\{\begin{array}{l}
t_{T x} \\
t_{T y} \\
t_{N}
\end{array}\right\} \quad\{t\}^{\text {bot }}=\left\{\begin{array}{l}
t_{T x} \\
t_{T y} \\
t_{N}
\end{array}\right\}^{\text {top }}
$$

and with this we can state the virtual internal work for each quadrilateral

$$
\begin{aligned}
\delta W_{I}^{\text {bottom }} & =\int_{0}^{\ell_{1}} \int_{0}^{\ell_{2}} \delta\{u\}^{\text {bottom } T}\{t\}^{\text {bottom }} d x d y \\
\delta W_{I}^{\text {top }} & =\int_{0}^{\ell_{1}} \int_{0}^{\ell_{2}} \delta\{u\}^{\text {top } T}\{t\}^{\text {top }} d x d y .
\end{aligned}
$$


In contact mechanics, one is interested in the relative displacement field $\{g\}$ of the contacting surfaces. This is expressed for zero thickness elements through the relative displacement between the top and the bottom quadrilateral,

$$
\{g\}=\{u\}^{\text {top }}-\{u\}^{\text {bottom }}=[H(\xi, \eta)]\left(\{u\}_{\text {nodal }}^{\text {top }}-\{u\}_{\text {nodal }}^{\text {bottom }}\right) .
$$

Furthermore, we know from Newton's third law that the traction vectors of the elements in contact must be equal in magnitude and opposite in direction,

$$
\{t\}=\{t\}^{\text {top }}=-\{t\}^{\text {bottom }} \text {. }
$$

We can now implement contact laws as incremented and linearized constitutive relations [9] between contact tractions and the relative displacement field,

$$
\Delta\{t\}=\left[\frac{\partial\{t\}}{\partial\{g\}}\right]_{t+\Delta t\{g\}^{(j-1)}} \Delta\{g\}={ }^{t+\Delta t}[C]_{\text {Tangential }}^{(j-1)} \Delta\{g\} .
$$

A description of different contact laws can be found in Gaul and Nitsche [10].

The virtual work of the contact tractions is given by the summation of the virtual internal work for each element, as given in equation (17) and equation (18):

$$
\delta W_{C}=\delta W_{I}^{\text {top }}+\delta W_{I}^{\text {bottom }}=\int_{0}^{\ell_{1}} \int_{0}^{\ell_{2}} \delta\{g\}^{T}\{t\} d x d y
$$

Implementing the contact law in the virtual work expression yields the tangential contact stiffness matrix for a relative displacement quadrilateral element,

$$
[K]_{\text {Tangential }}=\int_{0}^{\ell_{1}} \int_{0}^{\ell_{2}}[H(\xi, \eta)]^{T}{ }^{t+\Delta t}[C]_{\text {Tangential }}^{(j-1)}[H(\xi, \eta)] d x d y
$$

These integrals are evaluated by applying the isoparametric concept and using Gaussian quadrature scheme [8]. The full stiffness matrix for the eight node zero thickness element is composed of the stiffness matrix of the quadrilateral element

$$
[K]=\left[\begin{array}{rr}
{[K]_{\text {Tangential }}} & -[K]_{\text {Tangential }} \\
-[K]_{\text {Tangential }} & {[K]_{\text {Tangential }}}
\end{array}\right]
$$

The stiffness matrix $[K]$ is 12-times singular due to its composition, thereby causing 12 zero-energy modes. A zero-energy mode, or so-called hourglass mode, is a displacement mode that does not correspond to a rigid body motion, and it produces zero strain energy [8]. As zero thickness elements are always clamped between continuum elements, all 12 zero-energy modes are suppressed.

For applications of zero thickness elements in geomechanics, see, e.g., Beer [11] and for applications in model update procedures see Ahmadian et al. [12]. 


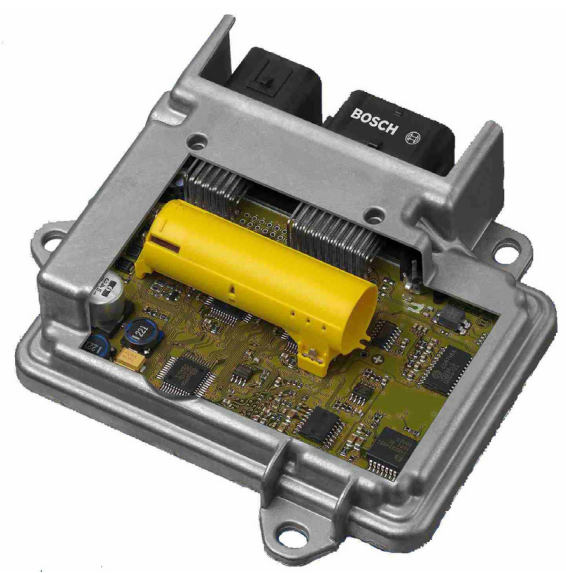

Figure 5: Airbag control unit generation 9 (Robert Bosch $\mathrm{GmbH}$ ). Source: http://www.bosch-presse.de/TBWebDB/bosch-pbj/de-DE/start.cfm
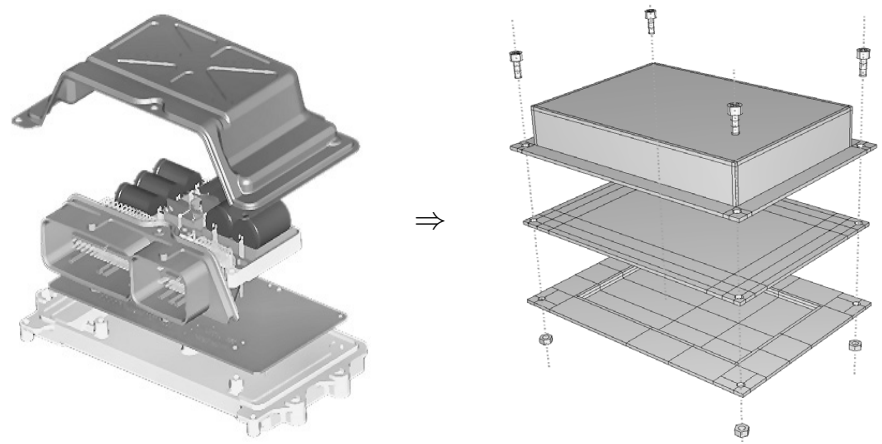

Figure 6: Exploded view of a control unit (left) and simplified test structure model (right) consisting of three simple aluminium parts connected by four bolts with nuts for investigating especially the influence of the contact region on the vibration behaviour.

\section{Application to a structure with bolted joints}

The contact model implemented in the presented zero thickness elements is applied to model the joint interfaces of a simplified control unit and thus predict the vibration behaviour. The simplified structure is depicted on the right of figure 6 .

The linear elastic material parameters of the aluminium parts are experimentally determined to minimize errors in the vibration simulation. The simulation itself consists of two steps. In a first preloading step the bolts and nuts are tightened causing a non-homogeneous contact pressure distribution in the contact interface 
which is depicted in figures 7 and 8 and by it a non-homogeneous contact stiffness distribution. Next, following the experimental approach, the impulse response of the structure is simulated and resonance frequencies and modal damping values are determined by modal analysis of the simulated impulse responses at different locations of the finite element mesh.

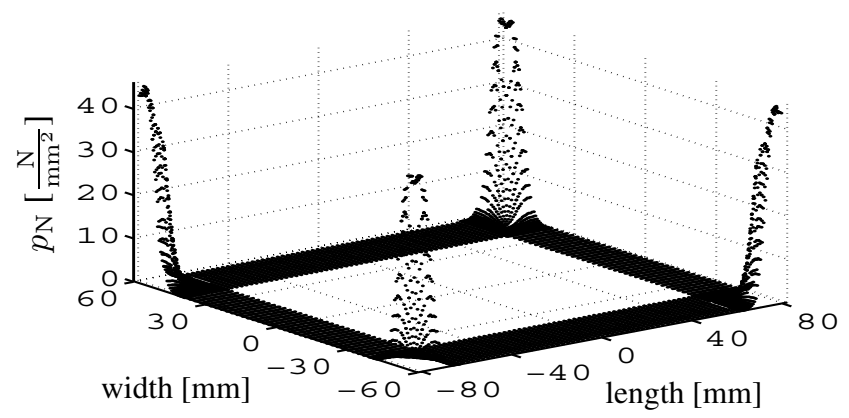

Figure 7: Normal pressure distribution $p_{\mathrm{N}}$ in the contact interface between upper cover and plate.

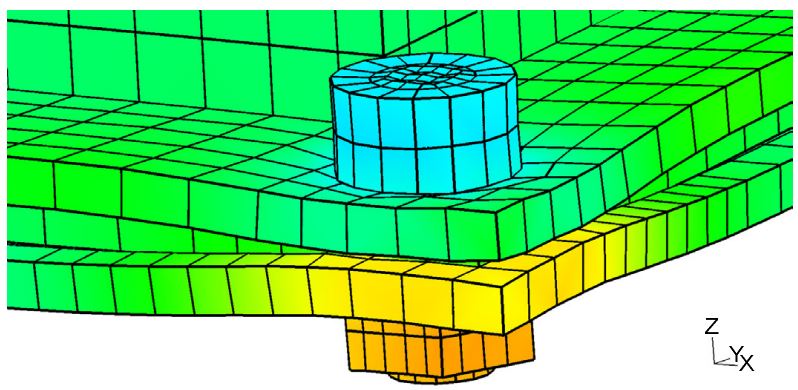

Figure 8: Gaping of the contact interfaces after applying the bolt load.

\subsection{Conclusion}

Table 1 compares simulated and measured results. Obviously, the new contact model accurately predicts the measured behaviour of this structure. 
Table 1: Comparison of resonance frequencies and modal damping values up to $2 \mathrm{kHz}$ determined by measuring the impulse response and following Experimental Modal Analysis (left) and by simulation of the impulse response, Fourier transform and following Modal Analysis (right).

\begin{tabular}{l|cc|cc}
\hline \hline & \multicolumn{2}{|c}{ Measured } & \multicolumn{2}{c}{ Simulated } \\
\hline Mode 1 & $889 \mathrm{~Hz}$ & $1.2 \%$ & $877 \mathrm{~Hz}$ & $0.9 \%$ \\
Mode 2 & $1101 \mathrm{~Hz}$ & $0.8 \%$ & $1113 \mathrm{~Hz}$ & $0.6 \%$ \\
Mode 3 & $1349 \mathrm{~Hz}$ & $1.1 \%$ & $1366 \mathrm{~Hz}$ & $0.7 \%$ \\
Mode 4 & $1424 \mathrm{~Hz}$ & $0.9 \%$ & $1386 \mathrm{~Hz}$ & $0.7 \%$ \\
Mode 5 & $1521 \mathrm{~Hz}$ & $0.7 \%$ & $1537 \mathrm{~Hz}$ & $0.5 \%$ \\
Mode 6 & $1645 \mathrm{~Hz}$ & $0.6 \%$ & $1660 \mathrm{~Hz}$ & $0.5 \%$ \\
Mode 7 & $1766 \mathrm{~Hz}$ & $0.7 \%$ & $1753 \mathrm{~Hz}$ & $0.6 \%$ \\
Mode 8 & $1960 \mathrm{~Hz}$ & $0.6 \%$ & $1982 \mathrm{~Hz}$ & $0.4 \%$ \\
\hline \hline
\end{tabular}

\section{References}

[1] Gaul, L., Albrecht, H. \& Wirnitzer, J., Semi-active friction damping of large space truss structures. Journal of Shock and Vibration, 11, pp. 173-186, 2004.

[2] Gaul, L. \& Lenz, J., Nonlinear dynamics of structures assembled by bolted joints. Acta Mechanica, 125, pp. 169-181, 1997.

[3] Johnson, K.L., Contact Mechanics. Cambridge University Press: Cambridge, 1985.

[4] Greenwood, J.A. \& Williamson, J.B.P., Contact of nominally flat surfaces. Proceedings of the Royal Society of London, Series A 295, pp. 300-319, 1966.

[5] Willner, K. \& Gaul, L., A penalty approach for contact description by fem based on interphase physics. Proceedings of Contact Mechanics II, eds. M.H. Aliabadi \& C. Allesandri, Computational Mechanics Publications: Southampton, pp. 257-264, 1995.

[6] Goodman, R.E., Taylor, R.L. \& Brekke, T.L., A model for the mechanics of jointed rock. Journal of the Soil Mechanics and Foundations Division, 94, pp. 637-660, 1968.

[7] Hohberg, J.M., A Joint Element for the Nonlinear Dynamic Analysis of Arch Dams. Birkhaeuser: Basel, 1992.

[8] Bathe, K.J., Finite Element Methods. Springer-Verlag: Berlin and Heidelberg, 2002.

[9] Wriggers, P., Computational Contact Mechanics. John Wiley \& Sons Ltd.: Chichester, 2002. 
[10] Gaul, L. \& Nitsche, R., The role of damping in mechanical joints. Applied Mechanics Reviews, 54, pp. 93-106, 2001.

[11] Beer, G., An isoparametric joint/interface element for finite element analysis. International Journal for Numerical Methods in Engineering, 21, pp. 585600, 1985.

[12] Ahmadian, H., Jalali, H., Mottershead, J. \& Friswell, M., Dynamic modeling of spot welds using thin layer interface theory. Proceedings of the Tenth Int. Congress on Sound and Vibration ICSV10, Stockholm, Sweden, pp. 34393446, 2003. 